\title{
Role of NADH: quinone oxidoreductase-1 in the tight junctions of colonic epithelial cells
}

\author{
Seung Taek Nam ${ }^{1, \#}$, Jung Hwan Hwang ${ }^{2, \#}$, Dae Hong Kim ${ }^{1}$, Mi Jung Park ${ }^{1}$, Ik Hwan Lee ${ }^{1}$, Hyo Jung Nam ${ }^{1}$, Jin Ku Kang ${ }^{1}$, \\ Sung Kuk Kim ${ }^{1}$, Jae Sam Hwang ${ }^{3}$, Hyo Kyun Chung , Minho Shong ${ }^{4}$, Chul-Ho Lee, ${ }^{2, *}$ E Ho Kim ${ }^{1, *}$ \\ ${ }^{1}$ Department of Life Science, College of Natural Science, Daejin University, Pocheon 487-711, ${ }^{2}$ Laboratory Animal Resource Center, Korea \\ Research Institute of Bioscience and Biotechnology (KRIBB), Daejeon 305-806, ${ }^{3}$ Department of Agricultural Biology, National Academy of \\ Agricultural Science, RDA, Suwon 441-707, ${ }^{4}$ Department of Internal Medicine, Chungnam National University, Daejon 301-721, Korea
}

\begin{abstract}
NADH:quinone oxidoreductase 1 (NQO1) is known to be involved in the regulation of energy synthesis and metabolism, and the functional studies of NQO1 have largely focused on metabolic disorders. Here, we show for the first time that compared to NQO1-WT mice, NQO1-KO mice exhibited a marked increase of permeability and spontaneous inflammation in the gut. In the DSS-induced colitis model, NQO1-KO mice showed more severe inflammatory responses than NQO1-WT mice. Interestingly, the transcript levels of claudin and occludin, the major tight junction molecules of gut epithelial cells, were significantly decreased in NQO1-KO mice. The colons of NQO1-KO mice also showed high levels of reactive oxygen species (ROS) and histone deacetylase (HDAC) activity, which are known to affect transcriptional regulation. Taken together, these novel findings indicate that NQO1 contributes to the barrier function of gut epithelial cells by regulating the transcription of tight junction molecules. [BMB Reports 2014; 47(9): 494-499]
\end{abstract}

\section{INTRODUCTION}

$\mathrm{NADH}$ :quinone oxidoreductase 1 (NQO1) catalyzes the reduction of quinone metabolites by using nicotinamide adenine dinucleotide $(\mathrm{NADH})$ as an electron donor $(1,2)$, thereby regu-

*Corresponding authors. Ho Kim, Tel: +82-31-539-1855; Fax: +82-31-539-1850; E-mail: hokim@daejin.ac.kr, Chul-Ho Lee, Tel: +82-42-860-4637; Fax: +82-42-860-4609; E-mail: chullee@kribb. re.kr

${ }^{\#}$ Nam, S. T., Hwang, J. H. contributed equally to this work as a first author.

http://dx.doi.org/10.5483/BMBRep.2014.47.9.196

Received 28 August 2013, Revised 4 October 2013, Accepted 19 December 2013

Keywords: Barrier dysfunction of epithelial cells, Chromosome condensation, Claudin-1, Gut epithelial cell tight junction, Gut inflammation, Histone acetylation/deacetylation, NQO1 knockout mice, Occludin, Transcription lating the intracellular ratio of NAD and NADH (two fundamental mediators of energy metabolism) in various cell systems. NQO1 is also known as an antioxidant flavoprotein that scavenges reactive oxygen species (ROS) (3). Having previously shown that NQO1 activity is associated with cancer (4) and metabolic disorders, including diabetes and obesity (5), we herein focused on the possible role of NQO1 in the gastrointestinal tract. We report for the first time that the expression levels of NQO1 are much higher in the intestine and colon than in other tissues. NQO1-KO (knockout) mice exhibited spontaneous gut inflammation, and in the DSS-induced colitis model, NQO1-KO mice showed more severe inflammatory symptoms than NQO1-WT (wild-type) mice.

Our results suggested that NQO1 could affect integrity of tight junction. Epithelial cells in the intestine and colon are known to form intercellular junctional complexes that act as a barrier, separating the lumenal contents from the human body $(6,7)$ and limiting the paracellular movement of various lumenal agents. Defects in this epithelial barrier have been proposed as a first step in a number of inflammatory bowel diseases (8). However, the molecular mechanisms responsible for regulating tight junction molecules are poorly understood. Here, we report for the first time that a macromolecular permeability study revealed that the tight junctions of epithelial cells were severely defective in NQO1-KO mice. This was due to the transcriptional downregulation of the tight junction proteins, claudin and occludin, and resulted in gut inflammation. Our results collectively suggest that NQO1 may be a key molecule responsible for regulating the epithelial cell tight junctions that form the physical barrier of the gut.

\section{RESULTS AND DISCUSSION}

NQO1 deficiency markedly increases gut permeability and inflammation

In the present study, we observed that the expression levels of NQO1 in the intestine and colon were relatively higher than those in other organs, including brain, spleen, liver and lung in NQO1-WT mice (Fig. 1A). Marked expression of NQO1 was also detected in the kidneys of NQO1-WT mice. This is con- 
sistent with the notion that NQO1 plays a critical role in the kidney, as suggested by the observation that pharmacological activation of NQO1 by $\beta$-lapachone can attenuate kidney injury (9). RT-PCR experiments confirmed that the transcript levels of NQO1 in the intestine and colon were significantly higher compared to those in the other tested organs (Fig. 1B). These results suggest that NQO1 may play a critical role in the gut.

Since one of the essential roles of gut epithelial cells is to form a physical barrier against lumenal contents, such as bacteria and orally ingested foods $(10,11)$, we assessed whether

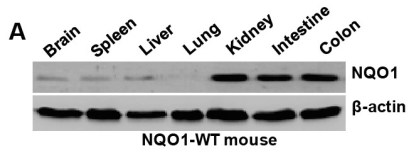

B
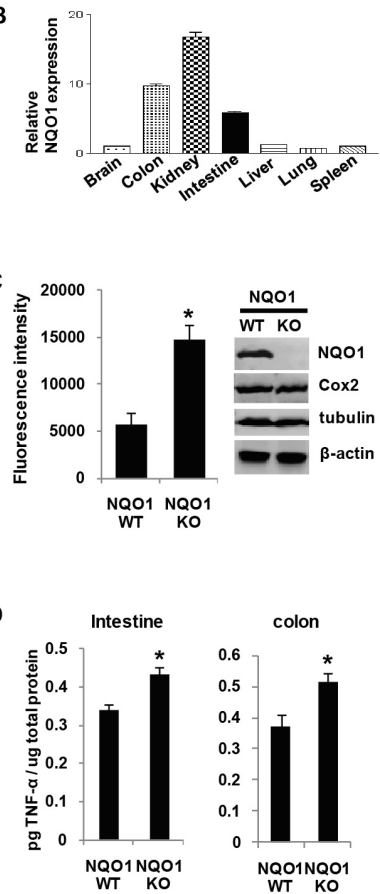

Fig. 1. NQO1 is abundant in gut epithelial cells, and NQO1 knockdown causes increased epithelial permeability in the mouse gut. (A) The organ distribution of NQO1 was elucidated by immunoblot analysis with tissue extracts of NQO1-WT mice. (B) The organ distribution of NQO1 was elucidated by RT-PCR with NQO1-specific primers. (C) Left panel: Ileal loops of NQO1-WT and NQO1-KO mice were lumenally injected with fluorescein-labeled dextran, and blood fluorescence was determined. *P $<$ 0.05 vs. NQO1-WT mice ( $n=12$ per group). Right panel: Colon tissues from near the cecum were isolated from NQO1-WT and NQO1-KO mice, total protein extracts were resolved on polyacrylamide gels, and the blotted membrane was probed with antibodies against NQO1, Cox2, tubulin, and $\beta$-actin. (D) The concentrations of TNF- $\alpha$ were measured in the intestine (left panel) and colon (right panel). The bars represent the mean \pm SEM of three independent experiments (*P $<0.005$ vs. NQO1-WT mice). the lack of NQO1 affected epithelial cell tight junctions. We used a gut macromolecular permeability test to measure epithelial cell tight junctions (12). As shown in Fig. 1C (left panel), we observed significantly higher gut permeability in NQO1-KO mice compared to NQO1-WT mice ( 3-fold). Unlike other proteins, Cox-2 and tubulin, NQO1 protein was not detected in NQO1-KO mice, proving specific NQO1 gene knockout (Fig. 1C, right panel). The concentrations of the proinflammatory cytokine, TNF- $\alpha$, in the intestine (left panel) and colon (right panel) were significantly higher in NQO1-KO mice than in NQO1-WT (Fig. 1D), suggesting the presence of increased inflammation in NQO1-KO mice. These results suggest that NQO1 deficiency causes a marked increase in the macromolecular permeability of the gut, and that this may be due to mild inflammation of the intestine and colon.

\section{NQO1 deficiency causes transcriptional down-regulation of claudin and occludin}

Given that NQO1-KO mice showed increased gut perme-

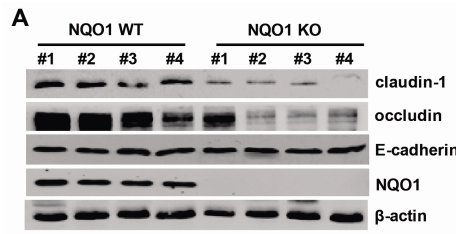

B

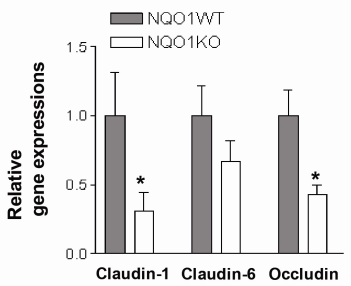

C

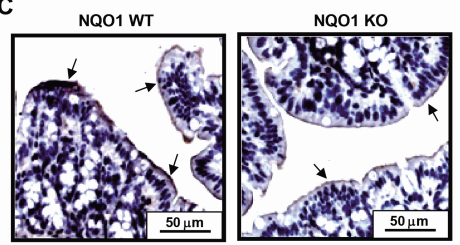

Fig. 2. Decreased expression of the tight junction molecules, occludin and claudin, in the colons of NQO1-KO mice. (A) Colonic tissue extracts were obtained from NQO1-WT and NQO1-KO mice respectively ( $n=8$ mice per group). The presented results are representative of three independent experiments. (B) The mRNA expressions levels of claudin-1, claudin- 6 and occludin were evaluated by real-time reverse transcription-PCR (RT-PCR). (C) Light micrographs of mouse colon samples subjected to immunohistochemistry for occludin (IHC stating; original magnification, $\times 200$ ). Data are representative of three independent samples (arrows indicate occludin proteins in the apical area of colonocytes). 
ability, we next assessed whether NQO1 deficiency affects the expression levels of tight junction molecules, such as claudin and occludin. Protein extracts were isolated from the colons of NQO1-WT and NQO1-KO mice, and the expression levels of claudin and occludin were determined by immunoblot analysis. As shown in Fig. 2A, the expression levels of claudin-1 and occludin in the colons of NQO1-KO mice were significantly lower than those in NQO1-WT colons. In contrast, the expression of E-cadherin, a major protein component of desmosomal junctions for cell-cell adhesion (12), did not differ between NQO-WT and NQO1-KO mice. RT-PCR experiments showed that the colons of NQO1-KO mice had reduced transcript levels of claudin-1, claudin- 6 and occludin compared to
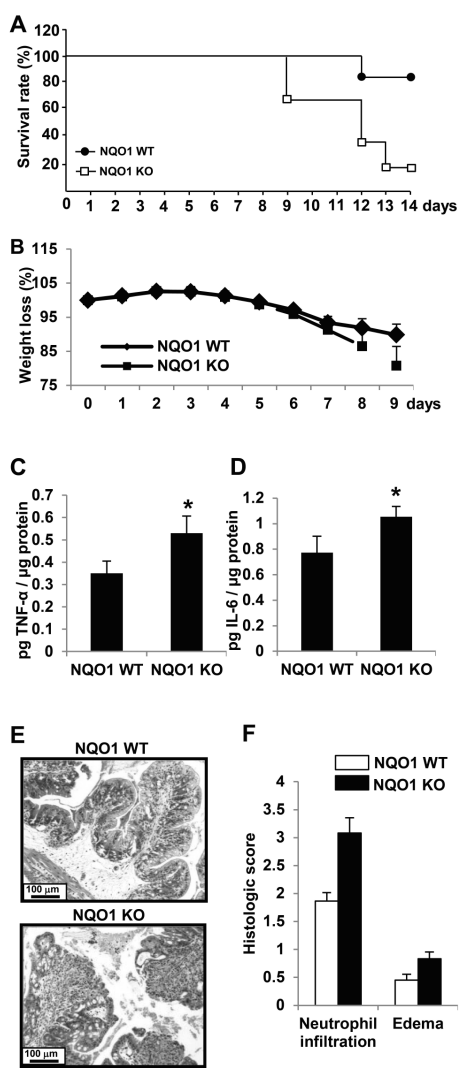

Fig. 3. NQO1 deficiency aggravates various symptoms of inflammation in DSS-induced colitis. (A) NOO1 knockdown significantly increases the mortality of DSS-treated mice. NQO1-WT and $-\mathrm{KO}$ mice received water containing 3\% DSS for the entire experimental period ( $n=12$ per group). (B) Marked weight loss was seen in DSS-treated NQO1-KO mice compared to NQO1-WT mice. (C and D) Mice were fed 3\% DSS-containing drinking water for 12 days; colonic tissues were collected; and the concentrations of TNF- $\alpha(C)$ and IL-6 (D) were measured. The bars represent the mean \pm SEM of three independent experiments, each with triplicate determinations; *indicates $\mathrm{P}<0.005$ vs. NQO1-WT mice. (E) Light micrographs of mouse colon samples (H\&E staining; original magnification, $\times 200$ ). (F) Histopathologic scoring for colonic inflammation. those of NQO1-WT mice (Fig. 2B). These results suggest that colonic NQO1 may be associated with the transcriptional regulation of tight junction molecules, thereby affecting epithelial barrier function. Immunohistochemistry confirmed that the protein levels of occludin were lower in the colonic epithelial cells of NQO1-KO mice versus NQO1-WT mice (Fig. 2C).

\section{NQO1 deficiency increases various symptoms of inflammation in the DSS-induced colitis model}

Next, we evaluated the potential pathophysiological role of NQO1 in compromised colons. To induce colitis in mice, NQO1-WT and NQO1-KO mice were treated with DSS (3\%) in their drinking water, and several clinical parameters of colitis (e.g., mortality and weight loss) were measured daily for the indicated durations. As shown in Fig. 3A, on day 14 of treatment, DSS-treated NQO1-KO mice showed significantly reduced survival rates, whereas DSS-treated NQO1-WT mice showed relatively weak reductions in survival by day 14 (NQO1-WT, 20\% vs. NQO1-KO, $80 \% ; n=12$ per group). Similarly, we observed marked decreases in body weight among NQO1-KO mice, but saw only a relatively small decrease in body weight among NQO1-WT mice (Fig. 3B). The levels of TNF- $\alpha$ (Fig. 3C) and IL-6 (Fig. 3D) were also higher in the colons of NQO1-KO mice compared to NQO1-WT mice. H\&E staining of colonic sections revealed that DSS-induced neutrophil infiltration were relatively higher in NQO1-KO mice compared to NQO1-WT mice (Fig. 3E). Next, we evaluated several histopathological parameters of colonic inflammation (13). Colonic tissue sections of NQO1-KO mice treated with DSS showed much higher histopathologic scores of colonic inflammation compared to NQO1-WT mice (Fig. 3F).

Given that DSS is known to cause severe paracellular permeability followed by massive exposure of lumenal pathogens to the human body, leading to gut inflammation and weight loss (14), the NQO1 deficiency (NQO1-KO)-induced increase in paracellular permeability and the DSS-induced mucosal damage may act synergistically to aggravate the inflammatory responses. Therefore, our results strongly support the physiological role of NQO1 in mediating tight junction integrity in the gut.

\section{Molecular mechanism for the transcriptional down-regulation of claudin and occludin in NQO1-KO mice}

Our findings suggest that the function of NQO1 in colonic epithelial cells may be associated with transcriptional regulation of the claudin and occludin genes. Having previously shown that Sp1 is a major transcriptional factor for claudin and occludin $(15,16)$, we assessed whether NQO1 knockdown affected the expression levels of Sp1. However, the expression level of Sp1 did not differ between NQO1-WT and NQO1-KO mice (Fig. 4A). The levels of acetylated histone-3 were significantly lower in the colons of NQO1-KO mice compared to NQO1-WT mice (Fig. 4A). However, total histone-3 levels did not differ between NQO1-WT and NQO1-KO mice, suggesting that the reduced acetylation of histone-3 could be associated with the transcrip- 
tional inhibitions of claudin and occludin in the colons of NQO1-KO mice. Since reduced histone acetylation has been shown to increase the folding of the histone-DNA complex, thereby inhibiting transcription (17-20), we measured the activity of histone deacetylases (HDACs) in nuclear extracts isolated from primary CECs of NQO1-WT and NQO1-KO mice. The purity of our subcellular fractionation was confirmed by immunoblot analysis with an $\beta$-actin antibody (data not shown). As shown in Fig. 4B, NQO1-KO mice exhibited significantly higher HDAC activity than NQO1-WT mice (Fig. 4B).

Consistent with previous reports showing that NQO1 largely functions as an antioxidant to decrease the formation of ROS, including $\mathrm{H}_{2} \mathrm{O}_{2}(21,22)$, we found that $\mathrm{H}_{2} \mathrm{O}_{2}$ levels were
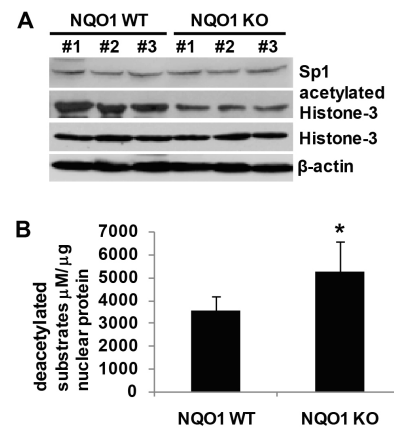

C
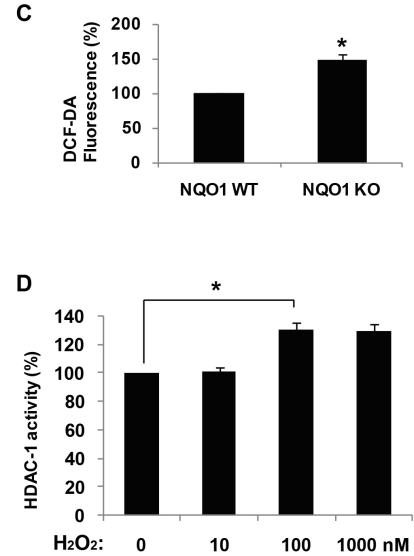

Fig. 4. In NQO1-KO mice, ROS accumulation sequentially causes HDAC activation, histone deacetylation, and transcriptional inhibition. (A) Colon samples taken from near the cecum were isolated from NQO1-WT and NQO1-KO mice ( $n=8$ per group), total protein extracts were resolved on polyacrylamide gels, and blots were probed with antibodies against Sp1, acetylated histone-3, histone-3, and $\beta$-actin. (B) Primary colonic epithelial cells were isolated from NQO1-WT and NQO1-KO mice, nuclear extracts were isolated and incubated with an acetylated fluorogenic substrate for $30 \mathrm{~min}$ at $37^{\circ} \mathrm{C}$, and fluorescence was measured $(* \mathrm{P}<0.05$ vs. NQO1-WT mice). (C) Intracellular ROS was determined by DCFH-DA. (D) Human active recombinant HDAC-1 proteins were incubated with an acetylated fluorogenic substrate in the presence of $\mathrm{H}_{2} \mathrm{O}_{2}$ for $30 \mathrm{~min}$ ( ${ }^{*} \mathrm{P}<0.005$ vs. untreated control). significantly higher in CECs from NQO1-KO mice compared to those of NQO1-WT mice (Fig. 4C). Next, we assessed whether these increased ROS levels could affect HDAC activity. To explore this, we incubated $1 \mu \mathrm{g}$ of active human recombinant HDAC-1 and substrate in the presence of different concentrations of $\mathrm{H}_{2} \mathrm{O}_{2}$, and then measured the activity of HDAC-1. As shown in Fig. 4D, $\mathrm{H}_{2} \mathrm{O}_{2}$ treatment significantly increased the activity of HDAC-1. Indeed, ROS (including $\mathrm{H}_{2} \mathrm{O}_{2}$ ) have been shown to increase the expression/activity of HDACs or their association with target proteins (e.g., p53 and FOXO3), leading to transcriptional regulation of target genes (23-26). These results suggest that HDACs are highly sensitive to NQO1-regulated ROS levels, and HDAC activity may affect the transcriptional regulation of certain genes. Thus, our present findings and the previous reports collectively suggest that knockdown of the well-known antioxidant, NQO1, sequentially triggers ROS accumulation, HDAC activation, histone deacetylation and chromatin compaction, and decreased transcription of target genes, such as claudin and occludin.

\section{MATERIALS AND METHODS}

\section{Mice and reagents}

NQO1-WT and NQO1-KO mice were kindly provided by Dr. Shong (Chungnam University, Daejeon, Korea). All mice were bred and maintained in conventional mouse facilities at Daejin University (Pochen, Korea), housed four per cage in a room maintained at a constant temperature $\left(25^{\circ} \mathrm{C}\right)$. All protocols conformed to the Animal Care and Use Committee guidelines. The polyclonal antibody against claudin- 1 was obtained from Cell Signaling Technology (Beverly, MA, USA). The polyclonal antibodies against NQO1, occludin, E-cadherin, acetylated histone-3, histone-3, Sp1, Cox-2 and tubulin were obtained from Santa Cruz Biotechnology (Santa Cruz, CA, USA). The $\beta$-actin antibody, dextran sulfate sodium salt (DSS) and 2, 7-dichlorofluorescin-diacetate (DCFH-DA) were purchased from Sigma Aldrich (St. Louis, MO, USA).

\section{Measurement of intestinal mucosal macromolecular permeability}

NQO1-WT and NQO1-KO mice were starved for $36 \mathrm{~h}$ prior to experiments, to reduce the luminal contents of their intestines. Each mouse was anesthetized with an intraperitoneal injection of Avertin (250 mg/kg; Sigma Aldrich). Both renal pedicles were ligated with 5-0 silk to prevent urinary excretion of the fluorescent probe. Ileal loops $(3-4 \mathrm{~cm})$ were also prepared by silk ligation, and then lumenally injected with normal saline $(0.3 \mathrm{ml}, \mathrm{PBS})$ containing fluorescein-labeled dextran (MW 4,000; 25 mg/ml; Sigma, Oakville, ON, Canada) using a 0.5 $\mathrm{ml} \mathrm{U}-100$ insulin syringe. To keep the animal warm and protect the dye from light exposure, each mouse was covered with an aluminum foil blanket. After $3 \mathrm{~h}, 0.5 \mathrm{ml}$ of blood was collected by cardiac stab. The blood was centrifuged at 5,000 rpm for $10 \mathrm{~min}$, and the supernatant was diluted 1:2 in PBS 
$(\mathrm{pH}$ 7.3). The concentration of fluorescein-labeled dextran was determined with a GloMax 20/20 (Promega, Madison, WI, USA), as previously described (12).

\section{RNA isolation and real time RT-PCR}

RNA was prepared using the TRIzol reagent (Life Technologies, Gaithersburg, MD, USA), and reverse transcription (RT) was performed as previously described (5). The resulting product $(1 \mu \mathrm{l})$ was amplified with primers specific to mouse NQO1 (sense, 5'-GGTTTACAGCATTGGCCACACT-3', and antisense, 5'-AACAGGCTGCTTGGAGCAAA-3'), mouse claudin-1 (sense, 5'-GTGCAAAGTCTTCGACTCCT-3' and antisense, 5'-GACACA AAGATTGCGATCAG-3'), mouse claudin-6 (sense, 5'-ATGTGG AAGGTGACCGCCT-3' and antisense, 5'-CCCTCCCACACCAT CTGG-3'), mouse occludin (sense, 5'-CCAGGCAGCGTGTTCCT3' and antisense, 5'-TTCTAAATAACAGTCACCTGAGGGC-3'). The PCR reactions were conducted with the optimal number of cycles $\left(94^{\circ} \mathrm{C}\right.$ for $1 \mathrm{~min}, 58^{\circ} \mathrm{C}$ for $1 \mathrm{~min}$, and $72^{\circ} \mathrm{C}$ for $\left.1 \mathrm{~min}\right)$.

\section{Isolation of mouse colonic epithelial cells}

Primary colonic epithelial cells (CECs) were isolated from 4- to 6-week-old NQO1-WT or NQO1-KO mice using the previously described procedures (27). Briefly, segments of colonic mucosa were washed and digested in DMEM containing $10 \mathrm{mg}$ Dispase I (Boehringer Mannheim Biochemicals, Mannheim, Germany), $50 \mathrm{mg} / \mathrm{ml}$ gentamicin, $50 \mathrm{U} / \mathrm{ml}$ penicillin, $50 \mathrm{mg} / \mathrm{ml}$ streptomycin, and $0.4 \%$ Bactrim for $90 \mathrm{~min}$ at $37^{\circ} \mathrm{C}$. The recovered cells were plated to tissue culture plates coated with a 1:1 solution of DMEM:Matrigel (Becton Dickinson, San Jose, CA). The purity of colonic epithelial cells was evaluated by cytokeratin staining.

\section{Isolation of nuclear proteins from primary CECs}

CECs were collected by centrifugation at $500 \times \mathrm{g}$ and resuspended in five volumes of $0.3 \mathrm{M}$ sucrose and $2 \%$ Tween- 40 in Buffer A [10 mM HEPES-potassium hydroxide $(\mathrm{KOH}), \mathrm{pH}$ 7.9, containing $10 \mathrm{mM} \mathrm{KCl}, 1.5 \mathrm{mM} \mathrm{MgCl}_{2}, 0.1 \mathrm{mM}$ EGTA, $0.5 \mathrm{mM}$ dithiothreitol (DTT), $0.5 \mathrm{mM}$ phenylmethylsulfonyl fluoride (PMSF), $2 \mu \mathrm{g} / \mathrm{ml}$ leupeptin, and $2 \mu \mathrm{g} / \mathrm{ml}$ pepstatin A]. The cells then were subjected to freezing, thawing, and gentle homogenization, and nuclei were isolated by centrifugation at 3,000 rpm in Buffer A. The precipitated pellets were lysed in Buffer A to isolate nuclear proteins, as previously described (28).

\section{Measurement of reactive oxygen species (ROS)}

Primary CECs were incubated for $1 \mathrm{~h}$ with $10 \mu \mathrm{M}$ DCFH-DA, which is cleaved to form DCFH and oxidized by oxygen free radicals to produce fluorescent DCF. Fluorescence intensity was analyzed with a Fluoroscan Ascent FL microplate reader (Thermo Fisher Scientific, Waltham, MA, USA) using 485 nm excitation and $538 \mathrm{~nm}$ emission filters.

\section{Enzymatic activity assay for HDACs}

HDAC activity was measured using a fluorescence activity as- say kit (Cayman Chemical, Ann Arbor, MI, USA) (28). Briefly, nuclear proteins $(1 \mu \mathrm{g})$ obtained from the primary CECs of NQO1-WT or NQO1-KO mice were incubated at $37^{\circ} \mathrm{C}$ with $100 \mu \mathrm{M}$ acetylated fluorogenic substrate in HDAC assay buffer. Fluorescence was measured using a Spectra Max M5 fluorescent plate reader (Molecular Devices, Sunnyvale, CA, USA) with excitation set at $360 \mathrm{~nm}$ and emission set at 460 nm. Active human recombinant HDAC-1 (Biomol, Palatine House, Matford Court, UK) was also used to measure deacetylase activity in the presence and absence of $\mathrm{H}_{2} \mathrm{O}_{2}$.

\section{Mouse colitis and clinical assessment of symptoms}

Acute colitis was induced by administration of dextran sodium sulfate (DSS, 40-50 kDa; ICN, Costa Mesa, CA, USA). NQO1WT and NQO1-KO mice were given 3\% DSS dissolved in regular tap water throughout the experimental period (up to 14 days). For the duration of the experiment, animals were observed three times per day for morbidity, mortality and weight (14).

\section{Measurement of mouse IL-6 and TNF- $\alpha$}

Mouse colons were homogenized (40 seconds) in PBS and centrifuged $\left(11,000 \times \mathrm{g}, 10 \mathrm{~min}\right.$ at $\left.4^{\circ} \mathrm{C}\right)$, and supernatants were collected. Mouse IL- 6 and TNF- $\alpha$ were measured by ELISA kits (R\&D Systems, Minneapolis, MN, USA) (14).

\section{Immunoblot analysis}

Mouse tissues were washed with cold PBS and lysed in buffer $(150 \mathrm{mM} \mathrm{NaCl}, 50 \mathrm{mM}$ Tris-HCl, pH 8.0, $5 \mathrm{mM}$ EDTA, $1 \%$ Nonidet P-40), and equal amounts of protein were fractionated by SDS-polyacrylamide gel electrophoresis (SDS-PAGE). The appropriate antibodies were applied, and antigen-antibody complexes were detected with the LumiGlo reagent (New England Biolabs, MA, USA) (14).

\section{Statistical analysis}

The results are presented as mean values \pm SEM. Data were analyzed using the SIGMA-STAT professional statistics software program (Jandel Scientific Software, San Rafael, CA, USA). Analyses of variance with protected $t$ tests were used for intergroup comparisons.

\section{ACKNOWLEDGEMENTS}

This work was supported by a grant from the Next-Generation BioGreen 21 Program (no, PJ008158), Rural Development Administration, and a grant from the National Research Foundation of Korea (NRF) funded by the Korean government (MSIP) (no, 2013R1A2A1A01011071), Republic of Korea.

\section{REFERENCES}

1. Berger, F., Ramirez-Hernandez, M. H., and Ziegler, M. (2004) The new life of a centenarian: signalling functions of $N A D(P)$. Trends Biochem. Sci. 29, 111-118. 
2. Pollak, N., Dolle, C., and Ziegler, M. (2007) The power to reduce: pyridine nucleotides-small molecules with a multitude of functions. Biochem. J. 402, 205-218.

3. Jaiswal, A. K. (2000) Regulation of genes encoding $\mathrm{NAD}(\mathrm{P}) \mathrm{H}$ : quinone oxidoreductases. Free Radic. Biol. Med. 29, 254-262.

4. Winski, S. L., Koutalos, Y., Bentley, D. L., and Ross, D. (2002) Subcellular localization of $\mathrm{NAD}(\mathrm{P}) \mathrm{H}$ :quinone oxidoreductase 1 in human cancer cells. Cancer Res. 62, 1420-1424.

5. Hwang, J. H., Kim, D. W., Jo, E. J., Kim, Y. K., Jo, Y. S., Park, J. H., Yoo, S. K., Park, M. K., Kwak, T. H., Kho, Y. L., Han, J., Choi, H. S., Lee, S. H., Kim, J. M., Lee, I., Kyung, T., Jang, C., Chung, J., Kweon, G. R. and Shong, M. (2009) Pharmacological stimulation of NADH oxidation ameliorates obesity and related phenotypes in mice. Diabetes 58, 965-974.

6. Nagumo, Y., Han, J., Bellila, A., Isoda, H., and Tanaka, T. (2008) Cofilin mediates tight-junction opening by redistributing actin and tight-junction proteins. Biochem. Biophys. Res. Commun. 377, 921-925.

7. Tokuda, S., Miyazaki, H., Nakajima, K., Yamada, T., and Marunaka, Y. (2010) $\mathrm{NaCl}$ flux between apical and basolateral side recruits claudin-1 to tight junction strands and regulates paracellular transport. Biochem. Biophys. Res. Commun. 393, 390-396.

8. Denizot, J., Sivignon, A., Barreau, F., Darcha, C., Chan, H. F., Stanners, C. P., Hofman, P., Darfeuille-Michaud, A., and Barnich, N. (2012) Adherent-invasive Escherichia coli induce claudin-2 expression and barrier defect in CEABAC10 mice and Crohn's disease patients. Inflamm. Bowel. Dis. 18, 294-304.

9. Oh, G. S., Kim, H. J., Choi, J. H., Shen, A., Choe, S. K., Karna, A., Lee, S. H., Jo, H. J., Yang, S. H., Kwak, T. H., Lee, C. H., Park, R. and So, H. S. (2014) Pharmacological activation of NQO1 increases NAD levels and attenuates cisplatin-mediated acute kidney injury in mice. Kidney Int. 85, 547-560.

10. Su, L., Shen, L., Clayburgh, D. R., Nalle, S. C., Sullivan, E. A., Meddings, J. B., Abraham, C., and Turner, J. R. (2009) Targeted epithelial tight junction dysfunction causes immune activation and contributes to development of experimental colitis. Gastroenterology 136, 551-563.

11. Schnoor, M., Betanzos, A., Weber, D. A., and Parkos, C. A. (2009) Guanylate-binding protein-1 is expressed at tight junctions of intestinal epithelial cells in response to interferon-gamma and regulates barrier function through effects on apoptosis. Mucosal Immunol. 2, 33-42.

12. Alscher, K. T., Phang, P. T., McDonald, T. E., and Walley, K. R. (2001) Enteral feeding decreases gut apoptosis, permeability, and lung inflammation during murine endotoxemia. Am. J. Physiol. Gastrointest. Liver Physiol. 281, G569-576.

13. Pothoulakis, C., Castagliuolo, I., LaMont, J. T., Jaffer, A., O'Keane, J. C., Snider, R. M., and Leeman, S. E. (1994) CP-96,345, a substance $P$ antagonist, inhibits rat intestinal responses to Clostridium difficile toxin A but not cholera toxin. Proc. Natl. Acad. Sci. U. S. A. 91, 947-951.

14. Rhee, S. H., Im, E., Riegler, M., Kokkotou, E., O'Brien, M., and Pothoulakis, C. (2005) Pathophysiological role of Toll-like receptor 5 engagement by bacterial flagellin in colonic inflammation. Proc. Natl. Acad. Sci. U. S. A. 102, 13610-13615.

15. Sade, H., Holloway, K., Romero, I. A., and Male, D. (2009)
Transcriptional control of occludin expression in vascular endothelia: regulation by Sp3 and YY1. Biochim Biophys. Acta 1789, 175-184.

16. Dufresne, J., and Cyr, D. G. (2007) Activation of an SP binding site is crucial for the expression of claudin 1 in rat epididymal principal cells. Biol. Reprod. 76, 825-832.

17. Richon, V. M., Sandhoff, T. W., Rifkind, R. A., and Marks, P. A. (2000) Histone deacetylase inhibitor selectively induces p21WAF1 expression and gene-associated histone acetylation. Proc. Natl. Acad. Sci. U. S. A. 97, 10014-10019.

18. Richon, V. M., Zhou, X., Rifkind, R. A., and Marks, P. A. (2001) Histone deacetylase inhibitors: development of suberoylanilide hydroxamic acid (SAHA) for the treatment of cancers. Blood Cells Mol. Dis. 27, 260-264.

19. Marks, P. A., Richon, V. M., Breslow, R., and Rifkind, R. A. (2001) Histone deacetylase inhibitors as new cancer drugs. Curr. Opin. Oncol. 13, 477-483.

20. Marks, P. A., Richon, V. M., and Rifkind, R. A. (2000) Histone deacetylase inhibitors: inducers of differentiation or apoptosis of transformed cells. J. Natl. Cancer Inst. 92, 1210-1216.

21. Begleiter, A., Hewitt, D., Maksymiuk, A. W., Ross, D. A., and Bird, R. P. (2006) A NAD(P)H:quinone oxidoreductase 1 polymorphism is a risk factor for human colon cancer. Cancer Epidemiol. Biomarkers. Prev. 15, 2422-2426.

22. Palming, J., Sjoholm, K., Jernas, M., Lystig, T. C., Gummesson, A., Romeo, S., Lonn, L., Lonn, M., Carlsson, B., and Carlsson, L. M. (2007) The expression of NAD(P)H:quinone oxidoreductase 1 is high in human adipose tissue, reduced by weight loss, and correlates with adiposity, insulin sensitivity, and markers of liver dysfunction. J. Clin. Endocrinol. Metab. 92, 2346-2352.

23. Kang, K. A., Zhang, R., Kim, G. Y., Bae, S. C., and Hyun, J. W. (2012) Epigenetic changes induced by oxidative stress in colorectal cancer cells: methylation of tumor suppressor RUNX3. Tumour Biol. 33, 403-412.

24. Ito, K., Hanazawa, T., Tomita, K., Barnes, P. J., and Adcock, I. M. (2004) Oxidative stress reduces histone deacetylase 2 activity and enhances IL-8 gene expression: role of tyrosine nitration. Biochem. Biophys. Res. Commun. 315, 240-245.

25. Rahman, I., Gilmour, P. S., Jimenez, L. A., and MacNee, W. (2002) Oxidative stress and TNF-alpha induce histone acetylation and NF-kappaB/AP-1 activation in alveolar epithelial cells: potential mechanism in gene transcription in lung inflammation. Mol. Cell. Biochem. 234, 239-248.

26. Miura, K., Taura, K., Kodama, Y., Schnabl, B., and Brenner, D. A. (2008) Hepatitis C virus-induced oxidative stress suppresses hepcidin expression through increased histone deacetylase activity. Hepatology 48, 1420-1429.

27. Baumgart, D. C., Olivier, W. A., Reya, T., Peritt, D., Rombeau, J. L., and Carding, S. R. (1998) Mechanisms of intestinal epithelial cell injury and colitis in interleukin 2 (IL2)-deficient mice. Cell Immunol. 187, 52-66.

28. Nam, H. J., Kang, J. K., Kim, S. K., Ahn, K. J., Seok, H., Park, S. J., Chang, J. S., Pothoulakis, C., Lamont, J. T., and Kim, H. (2012) Clostridium difficile toxin A decreases acetylation of tubulin, leading to microtubule depolymerization through activation of histone deacetylase 6 , and this mediates acute inflammation. J. Biol. Chem. 285, 32888-32896. 